\title{
Neural Components of Social Evaluation
}

\author{
William A. Cunningham and Marcia K. Johnson \\ Yale University
}

\author{
J. Chris Gatenby and John C. Gore \\ Vanderbilt University
}

\author{
Mahzarin R. Banaji \\ Harvard University
}

\begin{abstract}
Evaluative responses appear to involve 2 seemingly distinct sets of processes: those that are automatically activated and others that are more consciously controlled. Using functional magnetic resonance imaging, the authors investigated the brain systems associated with automatic and controlled evaluative processing. Participants made either evaluative (good-bad) or nonevaluative (past-present) judgments about famous names. Greater amygdala activity was observed for names rated as "bad" relative to those rated as "good," regardless of whether the task directly involved an evaluative judgment (good-bad) or not (past-present). Good-bad judgments resulted in greater medial and ventrolateral prefrontal cortex (PFC) activity than past-present judgments. Furthermore, there was greater ventrolateral PFC activity in good-bad judgments marked by greater ambivalence. Together, these findings indicate a neural distinction between processes engaged for automatic and controlled evaluation. Whereas automatic processes are sensitive to simple valence, controlled processes are sensitive to attitudinal complexity.
\end{abstract}

Arguably, among the most important cognitive processes are those that are involved in people's evaluation of their physical and social environment, including objects, persons, and events. The seemingly ordinary act of assigning valence-good and bad-is crucial for survival, guiding behavior toward or away from a significant object in the immediate environment or in anticipation of future rewards and punishments in goal attainment. It is not surprising then, that the brain systems that underlie these processes have been of considerable interest (see LeDoux, 1996). Nevertheless, there remains much to be done to fully understand how various component processes work independently and together to produce complex and coherent social evaluations.

William A. Cunningham and Marcia K. Johnson, Department of Psychology, Yale University; J. Chris Gatenby and John C. Gore, Vanderbilt University Institute of Imaging Science, Vanderbilt University; Mahzarin R. Banaji, Department of Psychology, Harvard University.

Portions of this research were presented at the 2002 meeting of the Society of Personality and Social Psychology, Savannah, Georgia, and at the 2002 meeting of the Cognitive Neuroscience Society, San Francisco, California. This research was supported by National Institute of Mental Health Grant MH-75672 and National Science Foundation Grant SBR9709924 to Mahzarin R. Banaji and by National Institutes of Health Grant MH-62196 to Marcia K. Johnson. This article is based on William A. Cunningham's doctoral dissertation supervised by Mahzarin R. Banaji and Marcia K. Johnson, and we thank the additional members of the dissertation committee for their thoughtful suggestions: Geoffrey Cohen, Peter Salovey, and Brian Scholl. For helpful comments on earlier versions of this article, we thank Anthony Freidas, Greg Walton, Eric Uhlmann, Joe Bates, Carol Raye, Karen Mitchell, and Christy Marshuetz.

Correspondence concerning this article should be addressed to William A. Cunningham, Department of Psychology, Yale University, P.O. Box 208205, New Haven, Connecticut 06520. E-mail: william .cunningham@yale.edu
To understand the affective and cognitive processes of evaluation, the present research brought together theory, research, and methods of social psychology and cognitive neuroscience to clarify the nature of social evaluation. Using functional neuroimaging to examine variation in brain activity as a function of perceiver intentions, the information processed, and the interaction between intention and information, we can begin to identify the distinct cognitive and affective processes that are involved in social evaluations. In particular, the present study used theoretical ideas and findings about attitude/evaluation and attitudinal ambivalence to investigate the neural mechanisms that give rise to them. Neuroimaging evidence provides convergent validity for theoretical constructs used to characterize social cognition, and also offers suggestions about the constraints on social cognitive models of evaluation. Conversely, the theoretical framework from social cognition is critical for interpreting neuroimaging data regarding social processes and for understanding and generating new hypotheses about brain function.

\section{The Social Cognition of Evaluation}

In the past 2 decades, the use of indirect measures of social cognition has led to the discovery that evaluations along a goodbad dimension can occur automatically, even in the absence of conscious intention or awareness. Building on latency-based semantic priming procedures, Fazio, Sanbonmatsu, Powell, and Kardes (1986) found that binary good-bad responses to stimulus words with strong positive and negative connotations (e.g., vomit, rainbow) were facilitated when preceded by brief presentations of evaluatively congruent stimuli. Participants were able to more quickly indicate that a target word (e.g., beautiful) had a good meaning when preceded by a prime word with good meaning (e.g., triumph) compared with a prime with bad meaning (e.g., murder). 
Similarly, brief presentations of evaluatively negative words speeded responses to immediately subsequent negative words. This effect occurs even when the prime and target are not semantically related, suggesting that the evaluative meaning of information is automatically activated independent of semantic meaning. Extending these findings, Bargh, Chaiken, Govender, and Pratto (1992) demonstrated the same evaluative facilitation effect for a task that required no explicit evaluation. Instead of having participants make evaluative (good-bad) judgments about target stimuli, Bargh et al. asked participants to pronounce evaluatively laden target words that were preceded by evaluatively congruent or incongruent primes. Even when participants only pronounced target words, Bargh et al. found that the evaluative congruence of prime and target facilitated participants' responses. Lastly, the automaticity of evaluation has been demonstrated in studies in which similar effects have been produced using subliminal primes (Draine \& Greenwald, 1998) to further reduce the likelihood of conscious reflection.

Evaluations that are consciously constructed may not have the same basis as evaluations that are activated automatically by cues in the immediate environment. Several models of attitude and affect (Devine, 1989; Greenwald \& Banaji, 1995; Johnson \& Multhaup, 1992) propose that there are at least two distinct systems that contribute to evaluation: automatic/perceptual and controlled/reflective sets of cognitive processes. Perceptual emotional processes are more primitive; they are relatively automatic responses to stimuli in the immediate environment. Reflective emotion is internally generated, typically involving controlled processing, and can operate in the absence of immediate environmental triggers. Evidence for distinctions in evaluative processing comes from numerous studies investigating attitudes toward social groups and individual members of those groups. When attitudes toward social groups are measured with both self-report measures and latency-based measures, self-reported attitudes toward, for example, African Americans, the elderly, and foreigners are substantially more positive than those detected by latency-based measures (Fazio, Jackson, Dunton, \& Williams, 1995; Rudman, Greenwald, Mellott, \& Schwartz, 1999).

Although attitudes may sometimes be directly retrieved from memory, it is often the case that consciously experienced evaluations are built online from multiple sources of information that are both activated automatically and retrieved in a more controlled fashion (Wilson \& Hodges, 1992). Furthermore, both positive and negative information may be activated regarding an attitude object, and as additional information is brought to bear during controlled/ reflective evaluation, this information needs to be weighed for relevance. Attitude theorists have noted that ambivalent attitudes - attitudes that contain both positive and negative components of evaluation-toward objects can exist (Breckler, 1994; Cacioppo \& Berntson, 1994). As an example from the current experiment, the idea of former President Bill Clinton may activate both positive and negative information that is selected or differentially weighed to make a binary good-bad judgment. Additional cognitive component processes are likely necessary to resolve such situations of attitudinal ambiguity. Thus, whereas automatic evaluative processes may be involved primarily in generating quick evaluative judgments, controlled evaluative processes may be involved in resolving evaluatively complex information.

\section{The Neuroscience of Evaluation}

Typically explored in the context of fear conditioning, numerous animal studies have shown that the amygdala - a small structure found in both hemispheres of the medial temporal lobe-is critical for emotional learning and the expression of such learning (for a review, see LeDoux, 2000). Recent work using neuroimaging has replicated the link between amygdala activation and fear conditioning in humans (LaBar, Gatenby, Gore, LeDoux, \& Phelps, 1998; Phelps et al., 2001). Interestingly, it appears that the amygdala is not only involved in associative learning of rewards and punishments but also in more abstract, conceptual representations of fear and threat. For instance, Phelps et al. (2001) told participants that they might receive mild shocks when particular visual stimuli were presented. Although no actual shock was administered, Phelps et al. found amygdala activity to stimuli that were verbally suggested to be threatening. Even more generally, amygdala activation was found to presented words with threatening connotations compared with neutral words (Isenberg et al., 1999).

Besides the processing of fear, the amygdala also appears to be involved in more general evaluation and valence detection. Imaging data have shown that the amygdala responds more strongly to unpleasant odors (Zald \& Pardo, 1997), tastes (Zald, Lee, Fluegel, \& Pardo, 1998), emotional pictures (Morris et al., 1996), and words (Tabert et al., 2001) than to corresponding pleasant or neutral items. Further, patients with bilateral amygdala damage have difficulty judging emotional facial expressions, especially negative emotions such as fear, anger, and disgust (Adolphs et al., 1999; Calder, Keane, Manes, Antoun, \& Young, 2000).

Current neuroimaging work has suggested that the amygdala is involved in the automatic processes of evaluation. For example, conscious awareness of a valenced stimulus does not appear to be necessary to produce amygdala activation. In a conceptual replication of previous research on supraliminal emotional face processing (Morris et al., 1996), Whalen et al. (1998) demonstrated that subliminal presentations of emotionally fearful faces led to significant amygdala activation. In addition, Morris, Ohman, and Dolan (1998) found, using both subliminal and supraliminal presentations, that after participants were classically conditioned to associate particular angry faces with an aversive stimulus, the amygdala showed greater activity to these conditioned faces than to control faces.

In a study of automatic race bias, Phelps et al. (2000) demonstrated that behavioral measures in White participants of automatic racial evaluation (i.e., the Implicit Association Test and startle eye blink) correlated with amygdala activation to Black faces relative to White faces, whereas a self-report measure of racial bias (i.e., the Modern Racism Scale) did not. Cunningham et al. (2003) using functional magnetic resonance imaging (fMRI) found significantly greater amygdala activation to subliminal presentations of Black faces relative to White faces. Thus, responses in the amygdala likely provide a quick and crude, unconsciously processed, affectively charged evaluation of the environment that prepares an organism for immediate action.

In contrast, the amygdala does not appear to be necessary for other, more controlled, aspects of evaluation. For instance, although patients with bilateral amygdala damage show deficits in fear conditioning, they are able to accurately report the valence of objects (Adolphs et al., 1999; Bechara et al., 1995). Thus, 
nonamygdala processes may allow patients with amygdala damage to learn about, judge, evaluate, and report valence. Several prefrontal regions appear to play a role in these additional evaluative processes (Damasio, 1994). At a general level, the prefrontal cortex (PFC) is involved in higher order cognition involving executive control and deliberative, conscious, or reflective processing (e.g., Duncan \& Owen, 2000; Johnson \& Reeder, 1997; Stuss \& Benson, 1984). Structures in the PFC are necessary to learn complex associations and may contribute to awareness of emotional feeling states (Lane et al., 1998). Neuroimaging experiments in which participants are asked to reflectively generate (Teasdale et al., 1999), monitor (Henson, Rugg, Shallice, Josephs, \& Dolan, 1999), anticipate (Porro et al., 2002), attribute (Paradiso et al., 1999), or report on (Gusnard, Akbudak, Shulman, \& Raichle, 2001) emotional states typically show heightened prefrontal, especially medial prefrontal, activation.

Prefrontal contributions to affective evaluation likely allow for flexible evaluations that can integrate information regarding current memories for past events, anticipation of future events (including rewards and punishments), contextual variables, higher order motivations, and personal values with information provided by more automatic perceptual evaluative systems (e.g., Johnson \& Multhaup, 1992). Moreover, these systems may be necessary when evaluations of objects must be updated as new information is learned. Patients with damage to the ventromedial PFC, although able to perform normally on many standard cognitive neuropsychological tests, have difficulty with tasks that require higher order affective processing. For instance, these patients have difficulty organizing future-goal-directed behavior, show diminished capacity to respond to punishment, present unrealistically positive evaluations of self, and display inappropriate emotional reactions (Damasio, Tranel, \& Damasio, 1990).

It is important to note that although the PFC may be involved in controlled aspects of evaluation, it is likely that areas of PFC also respond to evaluation at an automatic level. For instance, Kawasaki et al. (2001) demonstrated, using single neuron recordings, that valence is detected in human ventromedial and ventrolateral PFC (vlPFC) within $160 \mathrm{~ms}$ of stimulus presentation, a rapidness that suggests automatic evaluative processing. Thus, although the reflective processes that we discuss in this article likely involve PFC, the PFC is a heterogeneous area and as a whole should not be equated with only controlled processes. In addition, suggesting that the PFC is involved in more controlled-reflective aspects of evaluation and that the amygdala is involved in more automatic aspects of evaluation is not to suggest that the amygdala is impervious to conscious control (see Cunningham et al., 2003; Ochsner, Bunge, Gross, \& Gabrieli, 2002; Pessoa, McKenna, Gutierrez, \& Ungerleider, 2002; Schaefer et al., 2002) but rather that additional reflective processes are involved in the generation of more reflective or conscious evaluation.

\section{An Integration}

Research investigating the neural systems involved in evaluation has either been restricted to explorations of the automatic components of evaluation or has not explicitly tried to disentangle the automatic from the controlled processes of evaluation and attitude. However, one line of research has supported the idea that the amygdala and other subcortical structures are likely to be involved in automatic, unconscious evaluative processing, and some of the prefrontal contributions to evaluation may reflect more deliberate and controlled processing. Using a gambling task, Bechara, Damasio, Damasio, and Lee (1999) demonstrated that damage to the amygdala resulted in a lack of skin conductance when receiving a reward or punishment or while selecting stimuli with positive or negative consequences. In contrast, damage to the ventromedial PFC resulted in a lack of skin conductance only when selecting stimuli that have positive or negative consequences. This result suggests that the ventromedial PFC is involved in the anticipatory function of evaluation. That is, whereas the amygdala is involved in reactions to the valence of perceptual stimuli, the ventromedial PFC may be involved in reflective processes of evaluation, especially when immediate rewards and punishments are not present.

For the most part, research examining evaluative processing has relied on stimulus-driven designs where the neural activities to distinct positive versus negative stimuli are compared. A key limitation of such designs is that they do not allow investigators to distinguish the extent to which automatic and controlled processes are involved in judgments of the stimuli. Without manipulating evaluative intentions, it is not possible to discern whether differences in activity reflect intentional evaluative processes under the participants' conscious control or automatic evaluative processes that would have occurred regardless of task. In a study (Zysset, Huber, Ferstl, \& von Cramon, 2002) that compared activity during controlled evaluative versus nonevaluative judgments, the stimuli that were used differed across conditions. When the same stimuli do not serve in both conditions and are therefore not equated, it is unclear whether the effects obtained reflect processes of controlled evaluation or differences in other processes (such as automatic evaluation) that might be elicited by the differences inherent in stimuli.

The present study was designed to systematically distinguish brain activity associated with deliberate, intentional social evaluative judgment from that associated with more automatic social evaluative judgment to understand the component processes of evaluation. We used identical stimuli for evaluative and nonevaluative judgment conditions: Names of famous people (e.g., Adolf Hitler, Bill Cosby) were selected as stimuli that could be judged as good or bad (good-bad task; evaluative judgment) or judged to be historical or present day (past-present task; nonevaluative judgment). By holding name stimuli constant, brain activation associated with the automatic component of evaluation should be observed in both the evaluative and nonevaluative conditions. Thus, additional brain activation found for the goodbad task compared with the past-present task primarily reflects controlled evaluative processing, because automatic evaluative processing should remain constant. Moreover, using a nonevaluative task allowed us to (a) examine the brain regions associated with differences in activation to bad compared with good names when participants were not deliberately engaged in evaluation and (b) compare such activation to the differences found for when participants were attending to evaluation. Brain activity in which "good" and "bad" are differentiated to the same degree whether or not the intention to do so is present can provide strong evidence of automatic evaluation: The conscious goal of the perceiver is irrelevant to the processing. 
Lastly, we explored the role of controlled/reflective processes in resolving multiple competing evaluative representations. By examining brain activity as a function of ambivalence in both the evaluative and nonevaluative tasks, we can observe whether ambivalence is processed regardless of evaluative goals or whether additional processes are brought to bear to resolve ambivalence when evaluations are consciously constructed.

\section{Method}

Participants were shown a series of names of famous people during fMRI. In the evaluative condition, participants indicated whether names referred to "good" or "bad" people. In the nonevaluative condition, participants indicated whether names referred to historical ("past") figures or current ("present") figures. For example, Adolf Hitler would be classified by most if not all participants as bad in the evaluative task and as past in the nonevaluative task. Likewise, Bill Cosby would be classified by most people as good in the evaluative task and as present in the nonevaluative task. Unlike previous studies that have used between-subjects designs to look at differences between evaluative and nonevaluative processing (see Crites \& Cacioppo, 1998; Ito \& Cacioppo, 2000), we used a withinsubjects design to compare activity for automatic and controlled evaluation in the same brains.

\section{Participants}

Fifteen participants were paid for their participation. Participants reported no abnormal neurological history and had normal or corrected-tonormal vision. All participants provided informed consent. One participant was excluded for head motion greater than $2 \mathrm{~mm}$, and 2 additional participants were excluded for knowing the identity of fewer than $70 \%$ of the famous names presented. Twelve participants (mean age 22 years; 6 women) remained for all further analyses.

\section{fMRI Parameters}

All imaging was conducted with a GE 1.5T (General Electric Medical Systems Signa, Milwaukee, WI) scanner at the Yale Magnetic Resonance Imaging Research Center. To get whole brain functional coverage, 26 axial slices (slice thickness: $4.5 \mathrm{~mm}$, no skip) were prescribed parallel to the anterior commissure-posterior commissure (AC-PC) line, with the 11th slice centered on the AC-PC line. Functional images were acquired from inferior to superior using a single-shot gradient echoplanar pulse sequence (echo time $=30 \mathrm{~ms}$, repetition time $=2 \mathrm{~s}$, in-plane resolution $=3.75 \times 3.75 \mathrm{~mm}$, matrix size $=64 \times 64$, and field of view $=$ $24 \times 24 \mathrm{~cm})$.

\section{Procedure}

During fMRI, participants categorized famous names along one of two dimensions (good-bad or past-present) and indicated their categorization by making one of two button presses with their right hand. Using PsyScope for Macintosh (Cohen, MacWhinney, Flatt, \& Provost, 1993), stimuli were forward projected using an LCD and overhead projector onto a clear screen at the base of the MRI bore. A prism mirror positioned over the participants' eyes allowed them to view stimuli. All stimuli were presented in white letters against a black background.

For each trial, a cue indicated whether the trial required a good-bad or a past-present response for $500 \mathrm{~ms}$, immediately followed by a famous name for $1.5 \mathrm{~s}$. Between trials, a fixation cross remained on the screen for 2-6 s to space out trials. Response latency and response category were collected for each trial. To synchronize stimulus presentations with functional scanning, all trials were initiated by a trigger sent by the MRI scanner at the beginning of each scan. Each of four runs contained 24 of each trial type (evaluative task: bad names; evaluative task: good names; nonevaluative task: bad names; nonevaluative task: good names).

Because good-bad evaluations of target names are inherently subjective, after scanning, participants completed a questionnaire in which all names were rated along four dimensions. Participants indicated on a $0-9$ scale the extent to which the target names represented good or bad individuals. Separate ratings of good and bad were obtained so that a measure of ambivalence could be obtained (i.e., to disambiguate names that were high on both good and bad from those that were high on one and low on the other dimension). In addition, participants rated their degree of knowledge about each target person. Finally, they indicated whether the person was a historical (past) or current (present) figure. These ratings were used to conduct an idiographic sorting of the fMRI data. An analysis of participant responses indicated a high degree of evaluative (good-bad) interrater consistency $(\alpha=.98)$. With this high degree of consistency, analyses using normative instead of the idiographic ratings used here yielded nearly identical results.

\section{Preprocessing}

Data were corrected for slice acquisition time and motion using SPM99 (Friston et al., 1995), then transformed to conform to the SPM99 default Montreal Neurological Institute echoplanar imaging (MNI EPI) brain interpolated to $4 \times 4 \times 4 \mathrm{~mm}$. Functional data were smoothed using a $8-\mathrm{mm}$ full-width-half-maximum kernel, and a high-pass filter using SPM99 defaults removed effects of scanner drift. To additionally condition the data, a low-pass filter removed frequencies greater than $.17 \mathrm{~Hz}$, a cutoff that represents the frequency after which signals as a function of experimental effects are no longer expected.

\section{Data Analysis}

Data were analyzed using the general linear model as implemented by SPM99. A series of regressors ${ }^{2}$ was constructed to examine blood oxygen level dependent (BOLD) ${ }^{3}$ brain activity to each of the four trial types: evaluative bad, evaluative good, nonevaluative bad, nonevaluative good. Whether a particular name was assigned as a good or bad trial was determined by the rating participants provided after scanning. ${ }^{4}$ Trials in which a participant did not know the person were dropped in these analyses. On average, fewer than $10 \%$ of names were dropped for any given participant. Because of response latency differences between conditions (see below), we conducted a second set of analyses in which we additionally added response latency for each trial as a regression parameter to remove potential confounds resulting from amount of time needed to

\footnotetext{
${ }^{1}$ Although the use of a within-subjects design allowed for more powerful statistical comparisons of differences in brain function within the same person, it is possible that different findings could emerge if participants were never in an evaluative context. Further work should compare between-subjects with within-subjects designs to determine the extent to which an evaluative frame of mind carries over to automatic evaluative processing.

${ }^{2}$ Two regressors were used for each trial type: the expected brain signal following neural activity and then a derivative to model the onset of the neural response.

${ }^{3}$ After neural activity, a systematic change in magnetic signal occurs over a period of about $12 \mathrm{~s}$, with the greatest change typically being observed about 4-6 s after neural activity. Analyses show differences in this signal (the BOLD signal) as a function of condition.

${ }^{4}$ Idiosyncratic name ratings were not collected for the first 2 participants. Names were assigned to condition for these participants using button presses recorded for good-bad trials during fMRI scanning. In addition, these participants were not included in the analyses of ambivalence.
} 
Table 1

Significant Areas of Differences in Blood Oxygen Level Dependent Signal as a Function of Task or Stimulus

\begin{tabular}{lccrrrrr}
\hline Comparison (area of activation) & Hemisphere & BA & Size & $t$ & $x$ & $y$ & $z$ \\
\hline Good-bad minus past-present & & & & & & & \\
$\quad$ Medial PFC & $\mathrm{L}$ & 10 & 51 & 5.21 & 4 & 56 & 20 \\
$\quad$ Ventrolateral PFC & $\mathrm{R}$ & 47 & 22 & 5.49 & 44 & 32 & -12 \\
$\quad$ Anterior cingulate & $\mathrm{L}$ & 32 & 63 & 7.53 & -12 & 40 & 20 \\
Ambivalence (good-bad trials) & $\mathrm{R}$ & 47 & 24 & 7.97 & 44 & 20 & -8 \\
$\quad$ Ventrolateral PFC & & & & & & & \\
Bad minus good & $\mathrm{L}$ & & 8 & 4.05 & -20 & -4 & -16 \\
$\quad$ Amygdala & $\mathrm{R}$ & 45 & 16 & 10.18 & 48 & 24 & 16 \\
$\quad$ Ventrolateral PFC & & & & & & &
\end{tabular}

Note. All statistical comparisons are based on $d f=11$. BAs provided for activation in cortex. BA $=$ Brodmann's area; $t=$ maximal $t$ statistic for the statistical difference: $x, y$, and $z=$ the $3 \mathrm{D}$ coordinates of the activation within normalized Montreal Neurological Institute (MNI) space; PFC = prefrontal cortex; L = left; $\mathrm{R}=$ right.

perform the task. No substantive differences were found between these two sets of analyses. We report the analyses that include the response latency covariate.

Contrast maps were generated for each participant by comparing the estimated hemodynamic signal for (a) the evaluative versus nonevaluative conditions and (b) the good versus bad names. The first set of contrasts tested for task-related differences-that is, activity associated with the deliberate act of evaluation (or the converse). The second set tested for areas of activation that responded to the valence of the stimuli, regardless of task goals. Presumably, this second set of contrasts reflects relatively more automatic evaluative processing. Random-effects composite $t$ maps were generated by averaging these individual participant contrast maps. To reduce the possibility of Type I error because of multiple comparisons, regions of activation were defined as those areas in which 10 contiguous voxels were significant at $p<.001$. This was done for all regions except for the amygdala, an a priori region of interest from previous published work as well as data from our own lab. For the amygdala, an anatomic mask was created to analyze only voxels within this specified region, which also reduced the possibility of a Type I error. Because of the focused nature of this analysis and the relatively few number of voxels in the amygdala mask image to be analyzed, the criterion for amygdala activation was defined as 5 or more voxels significant at $p<.01$. $^{5}$

Using the idiosyncratic ratings of names provided by participants, we conducted a second series of analyses to examine processing related to attitudinal ambivalence (e.g., names for which participants indicated both positivity and negativity on the independent post-scan ratings of good and bad). We calculated ambivalence for each name for each participant using the gradual threshold model proposed by Priester and Petty (1996). These values were entered as continuous scores in the fMRI analysis. For each participant, and separately for the good-bad and past-present trials, areas of activation were identified that correlated with increases in ambivalence. Random effect $t$ maps were generated using these individual participant contrast maps as input and were thresholded at $p<.001$ (with 10 contiguous voxels).

Timelines for neural activity were generated using the SPM ROI toolbox (Poldrack, n.d.). Regions of activation were defined functionally from each of the contrast images. Timelines were estimated for each of the four conditions for each of the functionally defined regions of interest. Timelines for each condition were adjusted for the residual effects of the other conditions and were subjected to the default SPM99 high-pass filter.

\section{Results}

\section{Response Times}

Participants responded more quickly during fMRI for good-bad than past-present trials $(1,856 \mathrm{~ms}$ vs. $1,921 \mathrm{~ms}), F(1,11)=6.04$, $p<.05$, and more quickly for good names than for bad names $(1,849 \mathrm{~ms}$ vs. $1,927 \mathrm{~ms}), F(1,11)=9.32, p<.05$. Response times to ambivalent names (defined as the $25 \%$ most ambivalent names compared with the rest) were longer than nonambivalent names in the good-bad condition (1,921 ms vs. $1,734 \mathrm{~ms})$ but not the past-present condition $(1,844 \mathrm{~ms}$ vs. $1,846 \mathrm{~ms}), F(1,9)=39.12$, $p<.05$.

\section{Controlled Evaluation}

As can be seen in Table 1, there were three areas of activation identified in a contrast comparing evaluative (good-bad) with nonevaluative (past-present) activation-the medial PFC (mPFC), the vIPFC, and the anterior cingulate.

$m P F C$ (BA 10). As can be seen in the timelines presented in Figure 1, after stimulus presentation, activity increased in the good-bad task and decreased in the past-present task. In a recent meta-analysis on the neuroimaging of emotion, Phan, Wager, Taylor, and Liberzon (2002) found that the mPFC was involved in many aspects of affective processing, regardless of the valence and sensory modality of the triggering stimulus. Interestingly, though, we noted that whereas nearly $70 \%$ of the studies that involved reflectively generated emotion showed activation in the $\mathrm{mPFC}$, only about $45 \%$ of the studies that involved perceptually generated emotion showed activation in this region. Further evidence for a role of the mPFC in reflective affect/evaluation comes from work examining anticipatory anxiety (Simpson, Drevets, Snyder, Gusnard, \& Raichle, 2001). Anxiety can be contrasted with fear in that fear often is exhibited in the presence of a threatening stimulus,

\footnotetext{
5 The logic behind using more conservative statistical cutoffs for fMRI data than typically are used in behavioral studies (e.g., $p<.05)$ comes from the large number of statistical comparisons computed in analyzing fMRI data. To avoid Type I errors, two types of corrections are used: (a) a conservative alpha level for detecting significance and (b) requiring several adjacent voxels to reach this threshold. The logic is that if several adjacent voxels are significant, this activity is far less likely to have occurred by chance alone than if a single voxel is significant. Yet, using these more conservative criteria can lead to Type II errors. Thus, as in behavioral research, achieving a balance between Type I and Type II errors is desirable. Of course, replication is another way to achieve such balance.
} 
whereas anxiety is exhibited in the anticipation of a threatening stimulus. That reflective processing activates this region more than perceptual processing further implicates this region in controlled aspects of emotion and evaluation (Johnson \& Multhaup, 1992).

vlPFC (BA 47). In addition to $\mathrm{mPFC}$, an area of right vlPFC (inferior frontal cortex, BA 47) showed more activity for evaluative than nonevaluative judgments (Figure 2). In memory retrieval and monitoring, relevant information needs to be separated from or given greater weight than irrelevant information (e.g., Johnson, Hashtroudi, $\&$ Lindsay, 1993). Consistent with a proposed inhibitory function of the PFC (e.g., Hasher \& Zacks, 1988; Shimamura, 1995), ThompsonSchill, D'Esposito, Aguirre, and Farah (1997) suggested that the vlPFC is involved in inhibiting irrelevant information during memory tasks. Following this idea, our evaluative task may involve more complex selection than does the nonevaluative task.

If the greater vlPFC activation observed for the evaluative than nonevaluative trials is a function of the online weighing of evaluative information, then we should expect more vlPFC activation to occur on trials where people are most ambivalent (i.e., target names that elicit both positive and negative evaluation). As an example, names like Bill Clinton and Yasser Arafat often fell into the high ambivalent category, whereas Adolf Hitler and Mahatma Gandhi fell into the nonambivalent category (only negative and positive, respectively).

To examine the effects of attitudinal ambivalence, two sets of analyses were conducted. Names were idiosyncratically defined as ambivalent or not, such that $25 \%$ of names were classified as ambivalent. Figure 2B shows that activity in the region of right vlPFC identified in the previous analysis (good-bad minus pastpresent) was greatest for ambivalent names in the good-bad task.
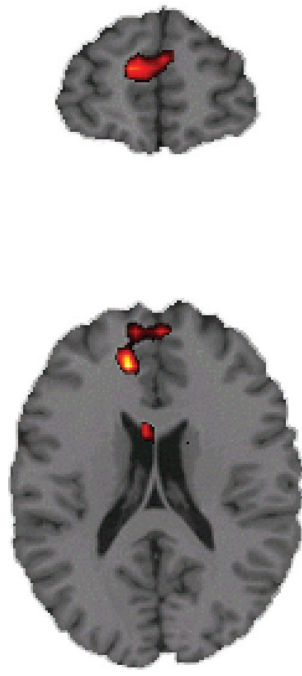

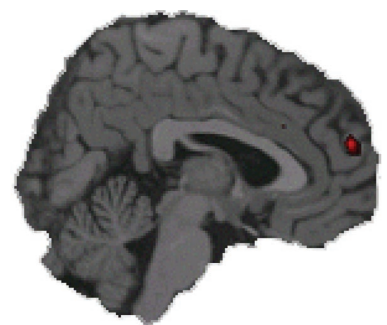

Good-Bad versus Past-Present

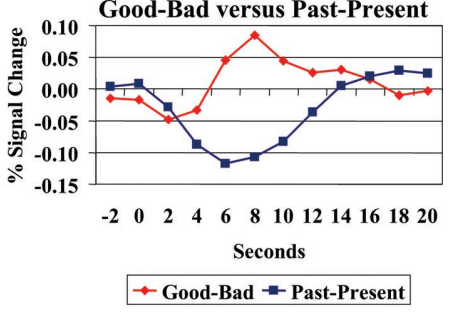

Figure 1. Random effects contrast map showing activation $(p<.001)$ for the good-bad minus past-present contrast in the medial prefrontal cortex (BA 10). Brain activation is presented in the coronal, sagittal, and axial planes for the location with the maximal activation difference between conditions (Montreal Neurological Institute [MNI] coordinates $[x, y$, $z]: 4,45,20)$. Timelines for both the good-bad and past-present tasks were generated from all significant voxels.
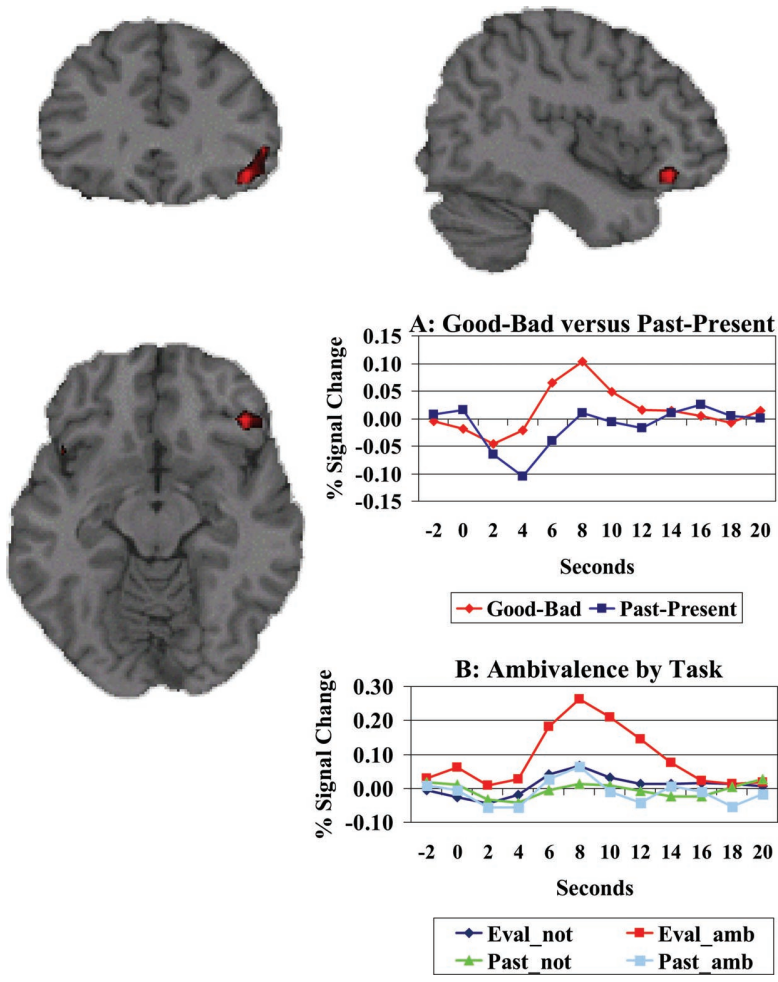

Figure 2. Random effects contrast map showing activation $(p<.001)$ for the good-bad minus past-present contrast in the right ventrolateral prefrontal cortex (BA 47). Brain activation is presented in the coronal, sagittal, and axial planes for the location with the maximal activation difference between conditions (Montreal Neurological Institute [MNI] coordinates $[x, y, z]: 44,32$, -12). Timelines for both the good-bad and past-present tasks were generated from all significant voxels (A). Additionally, timelines for these same voxels are presented separately for ambivalent and nonambivalent names for each task (B). For the purpose of the presentation, the top $25 \%$ of names for ambivalence are contrasted against the remainder of names. Eval_not = good-bad trials, nonambivalent names; Past_not $=$ past-present trials, nonambivalent names; Eval_amb = good-bad trials, ambivalent names; Past_ $\mathrm{amb}=$ past-present trials, ambivalent names.

In contrast, no difference between ambivalent and nonambivalent names was observed for the past-present task.

In the second analysis, we correlated the ambivalence score for each name for each participant with brain activity separately for the good-bad and past-present tasks. The only area that significantly correlated with ambivalence in the good-bad task was a region in right vlPFC. ${ }^{6}$ This region encompassed the region of vlPFC identified in the good-bad minus past-present contrast (Figure 2), but it was larger and included additional posterior regions of vlPFC. ${ }^{7}$ The correlation between ambivalence and vlPFC was significantly larger in the good-bad task than the

\footnotetext{
${ }^{6}$ When we dropped the threshold for detecting regions of activation to $p<.05$, we found a region of anterior cingulate cortex that was associated with greater ambivalence in the good-bad task.

${ }^{7}$ Time plots generated from the voxels significant in this analysis for ambivalent and nonambivalent names were virtually identical to those shown in Figure 2.
} 
past-present task, $F_{\text {interaction }}(1,9)=26.32, p<.001$. No area correlated with ambivalence in the past-present task.

\section{Good Versus Bad}

We compared brain activity with bad versus good names, both collapsing across task and analyzing within each task. As can be seen in Table 1 and Figures 3A and 4A, collapsing across task, greater bad than good activity was observed in the amygdala and in an area of right vlPFC (BA 45) that was located more superiorly than the one found to be sensitive to ambivalence.

Amygdala. In previous work, the amygdala has been shown to be more responsive to negative than positive information (Isenberg et al., 1999; Morris et al., 1996), though activation also has been observed to positive information relative to neutral information
(Hamann \& Mao, 2002). As can be seen in the timelines presented in Figure 3, we found amygdala activity for both good and bad names, with greater left amygdala activity for bad names than good names. Importantly, as can be seen in Figure 3, Panels B and C, this difference between good and bad names was observed in both the good-bad $(p<.01)$ and the past-present $(p<.01)$ tasks Moreover, the interaction between task and valence was not significant, $F_{\text {interaction }}(1,11)=0.01, p=.92$. The bad-good difference in amygdala activation was about the same magnitude whether or not participants consciously attended to the evaluative dimension, lending support to the idea that the amygdala is involved in the automatic processing of evaluation.

Right $v l P F C$ (BA 45). Mirroring the activity found for the amygdala, we found greater activation to bad names than good
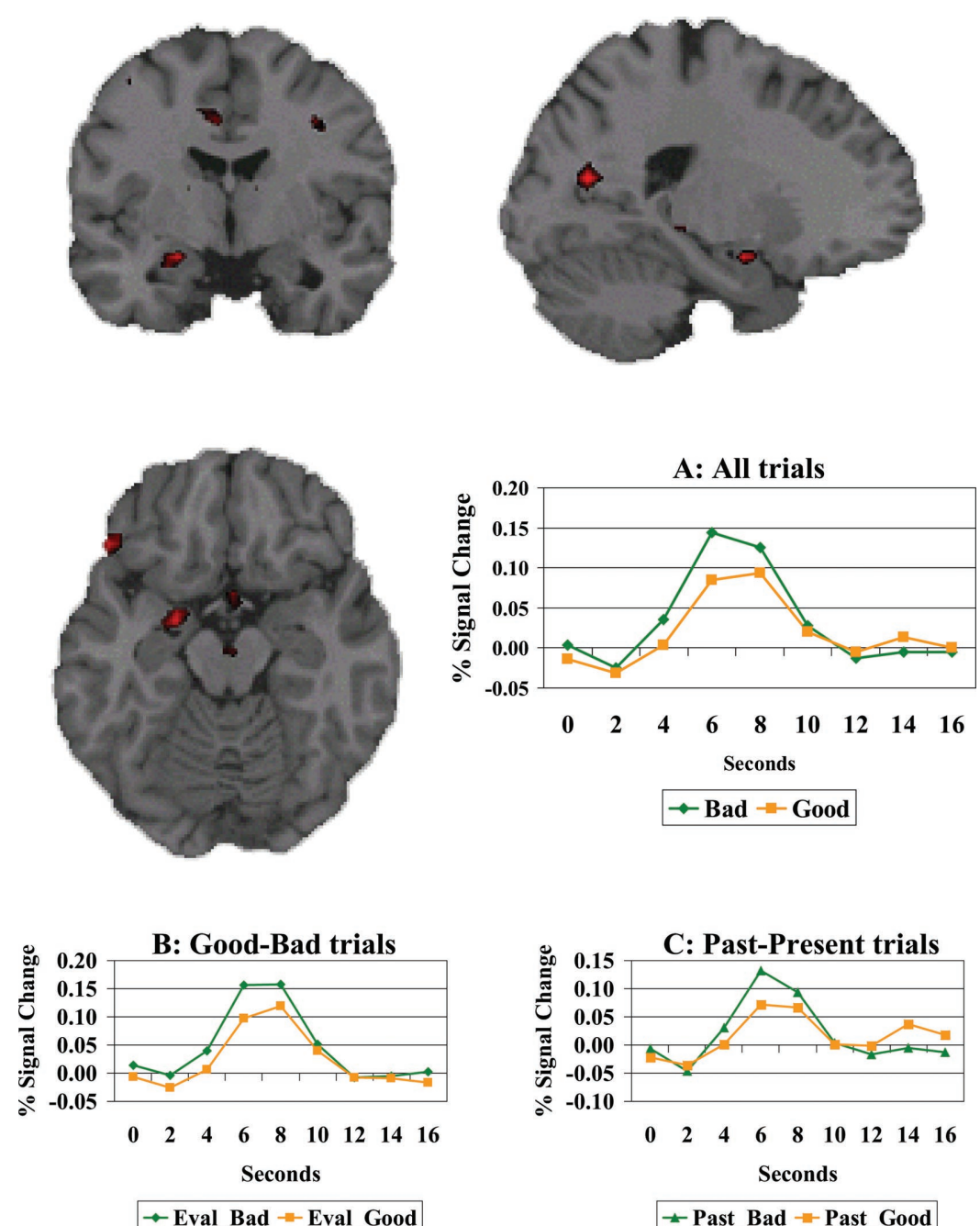

Figure 3. Random effects contrast map showing activation $(p<.01)$ for the bad-good contrast in the left amygdala. Brain activation is presented in the coronal, sagittal, and axial planes for the location with the maximal activation difference between conditions (Montreal Neurological Institute [MNI] coordinates $[x, y, z]:-20,-4$, $-16)$. Timelines plotted from significant voxels are presented for all trials, collapsing across task (A), and also separately for the good-bad (B) and past-present (C) trials. Eval_Bad = good-bad trials, bad names; Eval_Good $=$ good-bad trials, good names; Past_Bad = past-present trials, bad names; Past_Good = past-present trials, good names. 

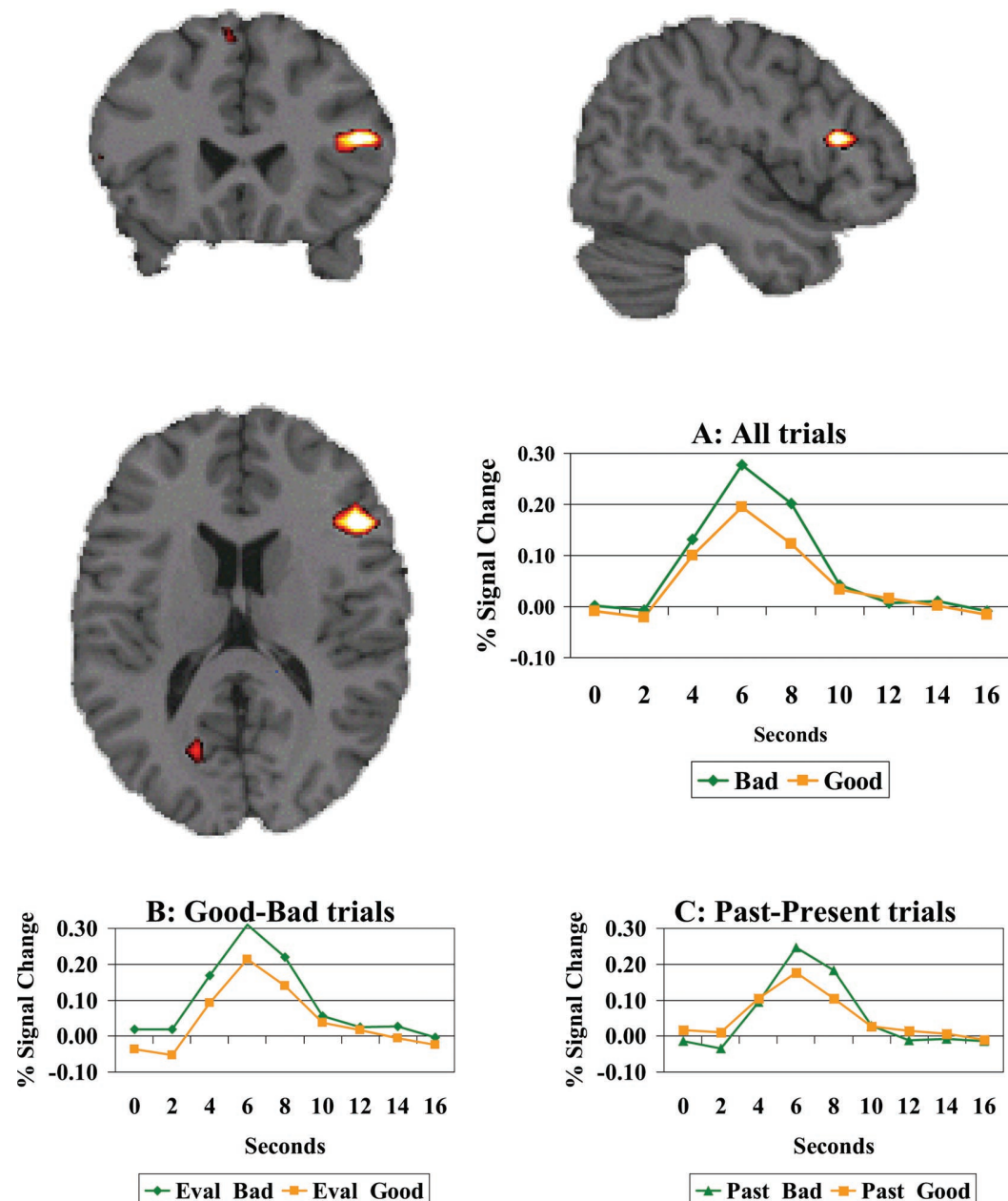

Figure 4. Random effects contrast map showing activation $(p<.001)$ for the bad-good contrast in the right inferior frontal cortex (BA 45). Brain activation is presented in the coronal, sagittal, and axial planes for the location with the maximal activation difference between conditions (Montreal Neurological Institute [MNI] coordinates $[x, y, z]:-48,24,16)$. Timelines plotted from significant voxels are presented for all trials, collapsing across task (A), and also separately for the good-bad (B) and past-present trials (C). Eval_Bad = good-bad trials, bad names; Eval_Good = good-bad trials, good names; Past_Bad = past-present trials, bad names; Past_Good = past-present trials, good names.

names in an area of right vlPFC, with no significant interaction between task and valence (Figure 4). In the study of affect and emotion, emotional reactions associated with withdrawal (e.g., fear) are often accompanied by greater prefrontal activation in the right than left hemisphere (Davidson \& Irwin, 1999; HarmonJones \& Allen, 1998; Sutton \& Davidson, 2000). Our data suggest a candidate region for this right laterality bias.

\section{Nonevaluative Processing}

Although not the main focus of this article, we note that several regions showed greater activation in the nonevaluative compared with the evaluative task, including areas of dorsolateral PFC and parietal cortex (areas that are associated with working memory and long-term memory processing). Both evaluative and nonevaluative judgments engaged these areas with the nonevaluative task recruiting them to a greater extent than the evaluative task.

\section{General Discussion}

Evaluative information is processed throughout all levels of the processing stream (see Cacioppo, Gardner, \& Berntson, 1999; Johnson \& Multhaup, 1992). Only hundreds of milliseconds after information is first perceived, the simple calculation of whether an object is good or bad is made, a calculation that is automatically used to direct attention and immediate behavior. In addition to these relatively simple automatic processes, human evolution has resulted in the ability to reflect about much more complex affective information. Interestingly, these evolutionarily newer reflective processes are not simply redundant with or replacements for older automatic evaluation processes. Rather, whereas automatic processes are quite well suited for processing simple valence, reflection allows for tackling evaluative information at other, presumably more complex, levels of analysis. Thus, the functional role of reflective/controlled evaluative processes may be to re- 
spond to and deal with evaluative complexity by withholding responses until more information is available, integrating multiple sources of information, and the deliberate retrieval of additional relevant memory representations. Consistent with this speculation, we found some brain regions sensitive to the simple good-bad valence of information regardless of intention to make an explicit evaluation and others sensitive to the complexity of information, but only when reflecting on evaluation.

Two regions were sensitive to the valence of the presented names: the amygdala and an area of right inferior frontal cortex (BA 45). Most interestingly, for both regions, we found greater activation to bad names than to good names whether or not participants were asked to evaluate those names. This finding is consistent with previous work on the automaticity of amygdala processing (see Morris et al., 1998; Whalen et al., 1998). From a functional perspective, it is interesting that although we found two regions that activated more for bad names than good names, we found no significant areas that activated more for good names than bad names. This automatic negativity bias may have arisen to aid survival in that as far as speeded judgments are concerned, it is far more consequential to mistake a bad object for a good one than vice versa.

It is important to note that such automatic evaluative processes are not immutable. Several recent studies have shown an important link between controlled processing and the inhibition or modulation of amygdala processing. For example, diverting attention (Pessoa et al., 2002), the activation of competing evaluative processes (Cunningham et al., 2003), maintaining an emotional response (Schaefer et al., 2002), or the controlled reappraisal of the stimulus (Ochsner et al., 2002) have been shown to modulate amygdala activity. Together, these data suggest that amygdala processes are specifically tuned for quick, automatic evaluation, and unless additional processes that intervene are recruited, such automatic processing will occur.

In addition to understanding automatic evaluation, the present research allows us to examine how social information is processed when people are in an evaluative frame of mind. One might hypothesize that the amygdala and right BA 45, regions that are sensitive to valence at an automatic level, should show heightened activity when attention is directed to evaluation at the controlled level. That is, there may be a common evaluative system that is additionally active during reflective evaluation. Yet this pattern of activation was not found in the current study. Although bad names activated these regions more so than good names in both tasks, we observed neither greater activity in these regions during deliberate evaluative processing compared with nonevaluative processing nor an interaction between task and valence. This pattern further suggests that the default orientation of the amygdala is for goodbad evaluations of the environment, providing a constant evaluative signal unless modified by other processes.

Given that the valence of names was detected equally in both the good-bad and past-present tasks and that the brain regions sensitive to valence do not show heightened activity during deliberate evaluation, an important question remains. What information is reflective evaluation sensitive to? That is, how does reflection aid evaluation in a way that automatic evaluative processing cannot accomplish?

We found that presumed controlled processes of evaluation were not sensitive to valence, but rather to the complexity of the information processed. As mentioned earlier, an important aspect of reflective evaluation is that it may take into account contextual variables, higher order ideals, and a multitude of other factors that can influence an evaluation. In addition, as more reflective processes are recruited, there is more opportunity to activate both positive and negative information regarding people, situations, and things. To the extent that this additional information requires more complex evaluations, this information needs to be sorted and weighed to generate an evaluation. Notably, we found activation in right vlPFC to be strongly associated with the processing of attitudinal complexity. The neural activity associated with ambivalent stimuli provides support for suggestions that conscious evaluations reflect a constructed online process rather than a controlled activation of a stored memory representation (see Wilson \& Hodges, 1992).

In addition, it is interesting to note that just as overall valence was not detected by any additional brain regions during the goodbad versus the past-present tasks, there were no correlates of ambivalence in the past-present task. This finding suggests that attitudinal ambivalence, or the state of conjoint positivity and negativity, is a state that arises during reflective processing. Thus, unlike the automatic attitude processes that are sensitive to overall valence to initiate quick approach or avoidance behaviors, controlled processes are sensitive to complexity of evaluative information. Given that the vlPFC is proposed to be involved in inhibitory processing, it is possible that in addition to selecting representations for the evaluation, these processes may also be involved in the inhibition of behavior or judgment until sufficient information is processed to make a more measured judgment. Thus, whereas the automatic system provides quick judgments, the controlled system may attempt to provide more accurate judgments.

We found that areas of $\mathrm{mPFC}$ were more active when making controlled evaluative compared with controlled nonevaluative judgments, a difference that was present equally for good and bad names and was not sensitive to ambivalence. Although the role of the $\mathrm{mPFC}$ in evaluation remains unclear, we can offer some speculations. As stated earlier, mPFC activity may be associated with a conscious, evaluative orientation or mode (cf. retrieval mode; Lepage, Ghaffar, Nyberg, \& Tulving, 2000) that may be a reflective analogue to the automatic processing mode of the amygdala. Yet a review of imaging studies in which mPFC activation has been found indicated that activation in this area is not specific to valenced evaluations (Cunningham, 2002). Studies that have required reflective social processing, broadly defined, also appear to have been associated with mPFC activity. Most notably, tasks that require making attributions of beliefs, intentions, and goals of others (Castelli, Happe, Frith, \& Frith, 2000) or self (Kelley et al., 2002) have activated the mPFC relative to control tasks. It is possible that the activity in this area reflects the attribution of mental states to others to determine culpability for particular behaviors. That is, for conscious evaluation, it may not be sufficient to know that A killed B. A reflective evaluation will likely involve assessing motives (e.g., why the person committed such an act) as well as the situational and other constraints on the behavior. Alternatively, mPFC may be engaged when tasks require coordinating multiple representations (i.e., are cognitively complex, Johnson \& Reeder, 1997), regardless of whether the task involves 
specifically social information. These alternatives remain to be explored.

In sum, the pattern of activation observed in the amygdala, in combination with the pattern observed in the PFC, echoed and substantiated at the neural level the proposed distinction between automatic and controlled evaluative processing. We found that separate brain systems were involved in the controlled-reflective aspects of evaluation as compared with those involved in the automatic-perceptual aspects. More importantly, we found that the automatic and controlled processes of evaluation were sensitive to different types of information. Brain areas associated with the automatic processing of evaluation were sensitive to simple valence information, whereas brain areas associated with the controlled processing of evaluation were sensitive to the attitudinal complexity of the name, that is, ambivalence. Together, these findings illustrate the ways in which long-standing theories of attitude and evaluation can be tested using a cognitive neuroscience approach. In addition to the convergent validity that such methods can provide, the workings of social processes can be examined online, thus having the potential to provide new insights into social behavior. Likewise, such analyses demonstrate the value of social psychological theories in understanding the workings of the brain as, in this case, it engages in making social decisions.

\section{References}

Adolphs, R., Tranel, D., Hamann, S., Young, A. W., Calder, A. J., Phelps, E. A., et al. (1999). Recognition of facial emotion in nine individuals with bilateral amygdala damage. Neuropsychologia, 37, 1111-1117.

Bargh, J. A., Chaiken, S., Govender, R., \& Pratto, F. (1992). The generality of the automatic attitude activation effect. Journal of Personality and Social Psychology, 62, 893-912.

Bechara, A., Damasio, H., Damasio, A. R., \& Lee, G. P. (1999). Different contributions of the human amygdala and ventromedial prefrontal cortex to decision-making. Journal of Neuroscience, 19, 5473-5481.

Bechara, A., Tranel, D., Damasio, H., Adolphs, R., Rockland, C., \& Damasio, A. R. (1995, August 25). Double dissociation of conditioning and declarative knowledge relative to the amygdala and hippocampus in humans. Science, 269, 1115-1118.

Breckler, S. J. (1994). A comparison of numerical indexes for measuring attitude ambivalence. Educational and Psychological Measurement, 54, 331-346.

Cacioppo, J. T., \& Berntson, G. (1994). Relationship between attitudes and evaluative space: A critical review, with emphasis on the separability of positive and negative substrates. Psychological Bulletin, 115, 401-423.

Cacioppo, J. T., Gardner, W. L., \& Berntson, G. G. (1999). The affect system has parallel and integrative processing components: Form follows function. Journal of Personality and Social Psychology, 76, 839855

Calder, A. J., Keane, J., Manes, F., Antoun, N., \& Young, A. W. (2000). Impaired recognition and experience of disgust following brain injury. Nature Neuroscience, 3, 1077-1078.

Castelli, F., Happe, F., Frith, U., \& Frith, C. (2000). Movement and mind: A functional imaging study of perception and interpretation of complex intentional movement patterns. Neuroimage, 12, 314-325.

Cohen, J. D., MacWhinney, B., Flatt, M., \& Provost, J. (1993). PsyScope: A new graphic interactive environment for designing psychology experiments. Behavioral Research Methods, Instruments, and Computers, 25, 257-271.

Crites, S. L., \& Cacioppo, J. T. (1996). Electrocortical differentiation of evaluative and nonevaluative categorizations. Psychological Science, 7 , $318-321$
Cunningham, W. A. (2002). The medial prefrontal cortex and affectivel social neuroscience: A review of 93 neuroimaging studies. Unpublished manuscript, Yale University.

Cunningham, W. A., Johnson, M. K., Raye, C. L., Gatenby, J. C., Gore, J. C., \& Banaji, M. R. (2003). An fMRI investigation of the conscious and unconscious evaluations of social groups. Unpublished manuscript, Yale University.

Damasio, A. R. (1994). Descartes' error: Emotion, reason, and the human brain. New York: Putnam.

Damasio, A. R., Tranel, D., \& Damasio, H. (1990). Individuals with sociopathic behavior caused by frontal damage fail to respond autonomically to social stimuli. Behavioural Brain Research, 41, 81-94.

Davidson, R. J., \& Irwin, W. (1999). The functional neuroanatomy of emotion and affective style. Trends in Cognitive Science, 3, 11-21.

Devine, P. G. (1989). Stereotypes and prejudice: Their automatic and controlled components. Journal of Personality and Social Psychology, 56, 5-18.

Draine, S. C., \& Greenwald, A. G. (1998). Replicable unconscious semantic priming. Journal of Experimental Psychology: General, 127, 286-303.

Duncan, J., \& Owen, A. W. (2000). Common regions of the human frontal lobe recruited by diverse cognitive demands. Trends in Cognitive Science, 23, 475-483.

Fazio, R. H., Jackson, J. R., Dunton, B. C., \& Williams, C. J. (1995). Variability in automatic activation as an unobtrusive measure of racial attitudes: A bona fide pipeline? Journal of Personality and Social Psychology, 69, 1013-1027.

Fazio, R. H., Sanbonmatsu, D. M., Powell, M. C., \& Kardes, F. R. (1986). On the automatic activation of attitudes. Journal of Personality and Social Psychology, 50, 229-238.

Friston, K. J., Holmes, A. P., Worsley, K. J., Poline, J. P., Frith, C. D., \& Frackowiak, R. S. J. (1995). Statistic parametric maps in functional imaging: A general linear approach. Human Brain Mapping, 2, 189-210.

Greenwald, A. G., \& Banaji, M. R. (1995). Implicit social cognition: Attitudes, self-esteem, and stereotypes. Psychological Review, 102, $4-27$.

Gusnard, D. A., Akbudak, E., Shulman, G. L., \& Raichle, M. E. (2001). Medial prefrontal cortex and self-referential mental activity: Relation to a default mode of brain function. Proceedings of the National Academy of Sciences, USA, 98, 4259-4264.

Hamann, S., \& Mao, H. (2002). Positive and negative emotional verbal stimuli elicit activity in the left amygdala. NeuroReport, 13, 15-19.

Harmon-Jones, E., \& Allen, J. J. B. (1998). Anger and frontal brain activity: EEG asymmetry consistent with approach motivation despite negative affective valence. Journal of Personality and Social Psychology, 74, 1310-1316.

Hasher, L., \& Zacks, R. T. (1988). Working memory, comprehension, and aging: A review and a new view. In G. H. Bower (Ed.), The psychology of learning and motivation: Advances in research and theory (Vol. 22, pp. 193-225). San Diego, CA: Academic Press.

Henson, R. N., Rugg, M. D., Shallice, T., Josephs, O., \& Dolan, R. J. (1999). Recollection and familiarity in recognition memory: An eventrelated functional magnetic resonance imaging study. Journal of Neuroscience, 19, 3962-3972.

Isenberg, N., Silbersweig, D., Engelien, A., Emmerich, S., Malavade, K., Beattie, B., et al. (1999). Linguistic threat activates the human amygdala. Proceedings of the National Academy of Sciences, USA, 96, 1045610459.

Ito, T. A., \& Cacioppo, J. T. (2000). Electrophysical evidence of implicit and explicit categorization processes. Journal of Experimental Social Psychology, 36, 660-676.

Johnson, M. K., Hashtroudi, S., \& Lindsay, D. S. (1993). Source monitoring. Psychological Bulletin, 114, 3-28.

Johnson, M. K., \& Multhaup, K. S. (1992). Emotion and MEM. In S. 
Christianson (Ed.), The handbook of emotion and memory: Research and theory (pp. 33-66). Hillsdale, NJ: Erlbaum.

Johnson, M. K., \& Reeder, J. A. (1997). Consciousness as meta-processing. In J. D. Cohen \& J. W. Schooler (Eds.), Scientific approaches to consciousness (pp. 261-293). Mahwah, NJ: Erlbaum.

Kawasaki, H., Kaufman, O., Damasio, H., Damasio, A. R., Granner, M., Bakken, H., et al. (2001). Single-neuron responses to emotional visual stimuli recorded in human ventral prefrontal cortex. Nature Neuroscience, 4, 15-16.

Kelley, W. M., Macrae, C. N., Wyland, C. L., Caglar, S., Inati, S., \& Heatherton, T. F. (2002). Finding the self? An event-related fMRI study. Journal of Cognitive Neuroscience, 14, 785-794.

LaBar, K. S., Gatenby, J. C., Gore, J. C., LeDoux, J. E., \& Phelps, E. A. (1998). Human amygdala activation during conditioned fear acquisition and extinction: A mixed-trial fMRI study. Neuron, 20, 937-945.

Lane, R. D., Reiman, E. M., Axelrod, B., Yun, L. S., Holmes, A., \& Schwartz, G. E. (1998). Neural correlates of levels of emotional awareness: Evidence of an interaction between emotion and attention in the anterior cingulate cortex. Journal of Cognitive Neuroscience, 10, 525535.

LeDoux, J. E. (1996). The emotional brain: The mysterious underpinnings of emotional life. New York: Simon \& Schuster.

LeDoux, J. E. (2000). Emotion circuits in the brain. Annual Review of Neuroscience, 23, 155-184.

Lepage, M., Ghaffar, O., Nyberg, L., \& Tulving, E. (2000). Prefrontal cortex and episodic memory retrieval mode. Proceedings of the National Academy of Sciences, USA, 97, 506-511.

Morris, J. S., Frith, C. D., Perrett, D. I., Rowland, D., Young, A. W., Calder, A. J., \& Dolan, R. J. (1996, October 31). A differential neural response in the human amygdala to fearful and happy facial expressions. Nature, 383, 812-815.

Morris, J. S., Ohman, A., \& Dolan, R. J. (1998, June 4). Conscious and unconscious emotional learning in the human amygdala. Nature, 393, 467-470.

Ochsner, K. N., Bunge, S. A., Gross, J. J., \& Gabrieli, J. D. E. (2002). Rethinking feelings: An fMRI study of the cognitive regulation of emotion. Journal of Cognitive Neuroscience, 14, 1215-1229.

Paradiso, S., Johnson, D. L., Andreasen, N. C., O’Leary, D. S., Watkins, G. L., Ponto, L. L., \& Hichwa, R. D. (1999). Cerebral blood flow changes associated with attribution of emotional valence to pleasant, unpleasant, and neutral visual stimuli in a PET study of normal subjects. American Journal of Psychiatry, 156, 1618-1629.

Pessoa, L., McKenna, M., Gutierrez, E., \& Ungerleider, L. G. (2002). Neural processing of emotional faces requires attention. Proceedings of the National Academy of Sciences, USA, 99, 11458-11463.

Phan, K. L., Wager, T., Taylor, S. F., \& Liberzon, I. (2002). Functional neuroanatomy of emotion: A meta-analysis of emotion activation studies in PET and fMRI. Neuroimage, 16, 331-348.

Phelps, E. A., O'Connor, K. J., Cunningham, W. A., Funayama, E. S., Gatenby, J. C., Gore, J. C., \& Banaji, M. R. (2000). Performance on indirect measures of race evaluation predicts amygdala activation. Journal of Cognitive Neuroscience, 12, 729-738.

Phelps E. A., O'Connor, K. J., Gatenby, J. C., Gore, J. C., Grillon C., \& Davis, M. (2001). Activation of the left amygdala to a cognitive representation of fear. Nature Neuroscience, 4, 437-441.

Poldrack, R. (n.d.). SPM ROI toolbox [Plug-in tool for region of interest analyses for the Statistical Parametric Mapping software package]. Retrieved from http://spm-toolbox.sourceforge.net

Porro, C. A., Baraldi, P., Pagnoni, G., Serafini, M., Facchin, P., Maieron,
M., \& Nichelli, P. (2002). Does anticipation of pain affect cortical nociceptive systems? Journal of Neuroscience, 22, 3206-3214.

Priester, J. R., \& Petty, R. E. (1996). The gradual threshold model of ambivalence: Relating the positive and negative bases of attitudes to subjective ambivalence. Journal of Personality and Social Psychology, 71, 431-449.

Rudman, L. A., Greenwald, A. G., Mellott, D. S., \& Schwartz, L. K. (1999). Measuring the automatic components of prejudice: Flexibility and generality of the Implicit Association Test. Social Cognition, 17, 437-465.

Schaefer, S. M., Jackson, D. C., Davison, R. J., Aguirre, G. K., Kimberg, D. Y., \& Thompson-Shill, S. L. (2002). Modulation of amygdalar activity by the conscious regulation of negative emotion. Journal of Cognitive Neuroscience, 14, 913-921.

Shimamura, A. P. (1995). Memory and the prefrontal cortex. In J. Grafman \& K. J. Holyoak (Eds.), Annals of the New York Academy of Sciences: Vol. 769. Structure and functions of the human prefrontal cortex (pp. 151-159). New York: New York Academy of Sciences.

Simpson, J. R., Drevets, W. C., Snyder, A. Z., Gusnard, D. A., \& Raichle, M. E. (2001). Emotion-induced changes in human medial prefrontal cortex: II. During anticipatory anxiety. Proceedings of the National Academy of Sciences, USA, 98, 688-693.

Stuss, D. T., \& Benson, D. F. (1984). Neuropsychological studies of the frontal lobes. Psychological Bulletin, 95, 3-28.

Sutton, S. K., \& Davidson, R. J. (2000). Prefrontal brain electrical asymmetry predicts the evaluation of affective stimuli. Neuropsychologia, 38 , 1723-1733

Tabert, M. H., Borod, J. C., Tang, C. Y., Lange, G., Wei, T. C., Johnson, R., et al. (2001). Differential amygdala activation during emotional decision and recognition memory tasks using unpleasant words: An fMRI study. Neuropsychologia, 39, 556-573.

Teasdale, J. D., Howard, R. J., Cox, S. G., Ha, Y., Brammer, M. J., Williams, S. C., \& Checkley, S. A. (1999). Functional MRI study of the cognitive generation of affect. American Journal of Psychiatry, 156, 209-215.

Thompson-Schill, S. L., D’Esposito, M., Aguirre, G. K., \& Farah, M. J. (1997). Role of left inferior prefrontal cortex in retrieval of semantic knowledge: A reevaluation. Proceedings of the National Academy of Sciences, USA, 94, 14792-14797.

Whalen, P. J., Rauch, S. L., Etcoff, N. L., McInerney, S. C., Lee, M. B., \& Jenike, M. A. (1998). Masked presentations of emotional facial expressions modulate amygdala activity without explicit knowledge. Journal of Neuroscience, 18, 411-418.

Wilson, T. D., \& Hodges, S. D. (1992). Attitudes as temporary constructions. In L. L. Martin \& A. Tesser (Eds.), The construction of social judgments (pp. 37-65). Hillsdale, NJ: Erlbaum.

Zald, D. H., Lee, J. T., Fluegel, K. W., \& Pardo, J. V. (1998). Aversive gustatory stimulation activates limbic circuits in humans. Brain, 121, 1143-1154.

Zald, D. H., \& Pardo, J. V. (1997). Emotion, olfaction, and the human amygdala: Amygdala activation during aversive olfactory stimulation. Proceedings of the National Academy of Sciences, USA, 94, 4119-4124.

Zysset, S., Huber, O., Ferstl, E., \& von Cramon, D. Y. (2002). The anterior frontomedian cortex and evaluative judgment: An fMRI study. Neuroimage, 15, 983-991.

Received September 25, 2002

Revision received May 22, 2003

Accepted May 22, 2003 